\title{
Exploring the role of general practitioners in addressing financial toxicity in cancer patients
}

\author{
Carla Thamm ${ }^{1,2} \odot$ - Jennifer Fox ${ }^{2} \cdot$ Nicolas H. Hart ${ }^{1,2,3,4} \cdot$ Joel Rhee $^{5} \cdot$ Bogda Koczwara $^{6,7}$. Jon Emery ${ }^{8,9}$. \\ Kristi Milley $^{8,9} \cdot$ Rebecca L. Nund $^{10} \cdot$ Raymond J. Chan ${ }^{1,2,11,12}$
}

Received: 25 February 2021 / Accepted: 6 July 2021 / Published online: 26 July 2021

(c) The Author(s), under exclusive licence to Springer-Verlag GmbH Germany, part of Springer Nature 2021

\begin{abstract}
Purpose Financial toxicity (FT) describes financial distress or hardship as an outcome of cancer and its treatment. Minimising the impact of FT requires early assessment and intervention. General practice plays a significant role in the support of a person with cancer and may have an important role in the management of FT. The purpose of this study was to understand perspectives of general practitioners (GP) on addressing FT in the primary care setting, which may then help inform strategies to further support collaborative efforts to address FT.

Methods A qualitative interpretive approach was utilised for this study. GPs were recruited through a GP conference and other professional networks using purposive, snowballing sampling techniques. Data collection continued until sufficient rich data had been obtained. Interviews were recorded and transcribed verbatim. The data were analysed using inductive analysis techniques.

Results Twenty $(n=20)$ GPs participated in semi-structured in-depth telephone interviews. GPs identified that their role positions them well to provide some FT support, but there are limitations. Perceptions and philosophies about cancer management were drivers of referrals and financial conversations. Priorities for care of FT by GPs included improved cost information provision and accessible support.

Conclusion GPs can play an important role in helping to address FT associated with cancer and its treatments if supported with the right information.
\end{abstract}

Keywords Cancer $\cdot$ Cancer patients $\cdot$ Financial toxicity $\cdot$ General practice $\cdot$ General practitioner

Carla Thamm

c.thamm@qut.edu.au

1 Cancer and Palliative Care Outcomes Centre, Queensland University of Technology, Brisbane, QLD, Australia

2 School of Nursing, Queensland University of Technology, Brisbane, QLD, Australia

3 School of Medical and Health Sciences, Edith Cowan University, Perth, WA, Australia

4 Institute for Health Research, University of Notre Dame Australia, Perth, WA, Australia

5 General Practice Academic Unit, School of Medicine, University of Wollongong, Wollongong, NSW, Australia

6 College of Medicine and Public Health, Flinders University, Adelaide, SA, Australia
7 Flinders Medical Centre, Adelaide, Australia

8 Primary Care Collaborative Cancer Clinical Trials Group (PC4), Carlton, Australia

9 Centre for Cancer Research and Department of General Practice, University of Melbourne, Melbourne, VIC, Australia

10 School of Health and Rehabilitation Sciences, University of Queensland, Brisbane, QLD, Australia

11 Division of Cancer Services, Princess Alexandra Hospital, Brisbane, QLD, Australia

12 Caring Futures Institute, College of Nursing and Health Sciences, Flinders University, Adelaide, SA, Australia 


\section{Introduction}

The diagnosis and treatment of cancer come with a substantial financial burden for the patient, their family, and the community [1-6] with the cascade of costs associated with cancer varying from diagnosis through to the end of treatment and beyond [7]. A recent systematic review identified this financial burden as significant in countries with private healthcare systems, universal public healthcare systems, or hybrid systems [8]. However, there could be differences related to political, sociodemographic, geographic, and cultural disparity. Thus, concerted efforts to manage the financial impact of cancer care on cancer patients and survivors are of utmost importance.

Financial toxicity (FT) is a term used to describe financial distress or hardship as an outcome of cancer treatment [9]. High levels of FT negatively affect quality of life (QoL) in cancer survivors, especially emotional well-being [10-14]. A recent systematic review including 25 studies of 271,732 cancer survivors reported the frequency of FT ranging from 28 to $48 \%$ using monetary measures such as percentage of household income and ranged from 16 to $73 \%$ using selfreport measures such as impacts on everyday living expenses [9]. Although FT has gained attention due to the high cost of drugs, it is now recognised that patients are affected by other costs associated with a cancer diagnosis such as transportation, absenteeism from work during treatment, supportive care, complementary, and alternative therapies [6]. A limited ability to return to work is also an ongoing issue $[6,15]$.

Minimising the impact of FT requires early assessment and intervention at the beginning of and during cancer treatment. Interventions include, but are not limited to, ensuring patients are effectively informed of their treatment options and implications for efficacy in a timely manner [1, 16]; screening for and assessing FT [1]; advocating on behalf of patients as required [1]; avoiding low-value treatment or care to minimise FT [1]; empowering patients through a return-to-work plan where appropriate [15]; and managing of emotional distress and other symptoms (e.g. pain, fatigue, neuropathy) related to FT or employment disruption [17]. Family members and communities are also a key part of the FT experience [1]. General practice, grounded in the qualities of person centredness, comprehensiveness, continuity, trusted, and enduring patient relationships [18], plays a significant role in the support of a person with cancer and may have an important role in management of FT [19]. Despite this, there has been little research into the potential role of GPs in supporting FT in cancer patients. Therefore, the aim of this study was to understand GP's perspectives on their role in addressing FT amongst cancer patients in the primary care setting. This understanding may then help inform strategies to further support collaborative efforts to address FT.

\section{Methods}

This study used a qualitative interpretive approach. Grounded theory methods were used to encourage the collection of rich data in an area of inquiry that had previously been unexplored [20]. Inductive analysis techniques used in grounded theory encourage analysis to be iterative and ongoing and commence from the beginning of data collection [20].

\section{Participants and recruitment}

GPs were recruited using purposive sampling between November 2019 and June 2020 from a General Practice Conference (Royal Australian College of General Practitioners, GP19) and through professional primary care networks. This technique ensured diverse levels of experience, and geographical locations were included. Snowballing techniques were also used after interviews had taken place, by asking if participants had colleagues they felt could contribute valuable insights into the study. This study was approved by the Queensland University of Technology human research ethics committee (1900000798).

\section{Data collection}

Semi-structured telephone interviews were conducted by the lead researcher (CT). Interviews were recorded and transcribed verbatim by a professional transcription service. An interview guide based on study aims and previous research was used (Table 1). Prompting questions were added to elicit more in-depth responses. In line with principles of qualitative research, interview questions were used as a guide and questions and conversations evolved throughout the interview and data collection period [21].

\section{Data analysis}

Two members of the research team (CT, JF) separately engaged with and analysed the data. During data collection, interview summaries and notes were made following each interview that allowed exploration of important ideas that arose in preceding interviews and facilitated early and ongoing interpretations of the data. Transcripts were coded inductively, employing open coding on paper to organise and categorise the data. Regular research meetings (CT, JF) facilitated review and discussion of alternative interpretations, groupings of categories, and interrelations between categories. During this stage, overarching themes were identified. Subthemes were developed to enhance the findings. Data collection continued throughout this period until the researchers ascertained that sufficient rich data 
Table 1 Interview guide

\begin{tabular}{|c|c|}
\hline Interview questions & Prompts \\
\hline - Have you heard of the term financial toxicity? & - Is it something you observe in cancer patients? \\
\hline $\begin{array}{l}\text { Thinking about when you are seeing a person who has just been } \\
\text { diagnosed with cancer: } \\
\text { - What are your perceptions of the financial concerns they may present } \\
\text { with? } \\
\text { - What do you feel is your role as a GP to support this? }\end{array}$ & $\begin{array}{l}\text { - What cost considerations would you consider when you discuss diag- } \\
\text { nostic and treatment options with patients? } \\
\text { - How do you advise patients as to whether patients should select public } \\
\text { versus private services? }\end{array}$ \\
\hline $\begin{array}{l}\text { Thinking about when you care for people at other stages of their } \\
\text { cancer journey, i.e. when a patient is undergoing cancer treat- } \\
\text { ment or after treatment completion (and the palliative care } \\
\text { phase): } \\
\text { - What are some of the cost considerations that your patients might } \\
\text { present to you throughout these times? } \\
\text { - What do you feel your role as a GP might be in supporting this? }\end{array}$ & $\begin{array}{l}\text { - Would there be any differences in your consideration and advice } \\
\text { depending on the phases of disease (during treatment vs. survivorship } \\
\text { vs. palliative care)? } \\
\text { - What would some of these differences be? }\end{array}$ \\
\hline $\begin{array}{l}\text { - Are there ways the cancer community can better support GP's to } \\
\text { manage their conversations about financial issues with their patients? }\end{array}$ & $\begin{array}{l}\text { - Are there tools or support roles that may help? } \\
\text { - Do you feel there are any other professionals that may be able to assist } \\
\text { you to support financial concerns in the primary care setting? }\end{array}$ \\
\hline
\end{tabular}

Table 2 Characteristics of general practitioner participants

\begin{tabular}{ll}
\hline Characteristics & $\begin{array}{l}\text { Sample } \\
(n=20)\end{array}$ \\
\hline GP experience (years) & \\
- <5 years & 3 \\
- 5-10 years & 4 \\
- 10-20 years & 5 \\
- 20-30 years & 4 \\
- 30-40 years & 3 \\
- >40 years & 1 \\
Practice location & \\
- Metropolitan & 13 \\
- Outer metro/semi-rural & 4 \\
- Rural/remote & 3 \\
\hline
\end{tabular}

with no new themes and a depth of understanding on the topic had been achieved.

\section{Results}

Twenty $(n=20)$ participants were in three states of Australia, across metropolitan, outer-metropolitan, semirural, rural, or remote settings. Years of experience as a GP varied from $<5$ to $>40$ years (Table 2). Interviews ranged from 15 to $45 \mathrm{~min}$. Data analysis yielded three main themes including role of GP; perceptions and philosophies of cancer management; and priorities for care. Eight subthemes were formulated from the overarching themes see Fig. 1.

\section{Role of the GP}

The first theme explored the positioning of the GP in being able to support FT.

\section{Traditional role}

GPs identified their core business is to care for their patients beyond the disease process:

... there is a responsibility there in terms of it is something that's impacting on your patient's wellbeing... the GP needs to be aware and front-and-center with that sort of thing. (GP9)

Further, their role as a 'a trusted information provider' (GP15) and 'care coordinator and advocate for the patient' (GP16) put them in a favourable position to address the FT of cancer patients. Long-term relationships can also result in patients confiding with their GP about issues beyond their cancer, including financial concerns:

If the relationship is longstanding or there's a fair bit of trust, I'll find the patients, even if they don't need to, will actually come in during their treatments [to discuss financial stress]. (GP17)

GPs also identified that their experience with managing mental health was something that situated them well to manage the psychological and psychosocial effects that FT can impose.

\section{Level of involvement in supporting FT}

In contrast to the positive reinforcement for the role of a GP in addressing the financial burden of cancer patients, 


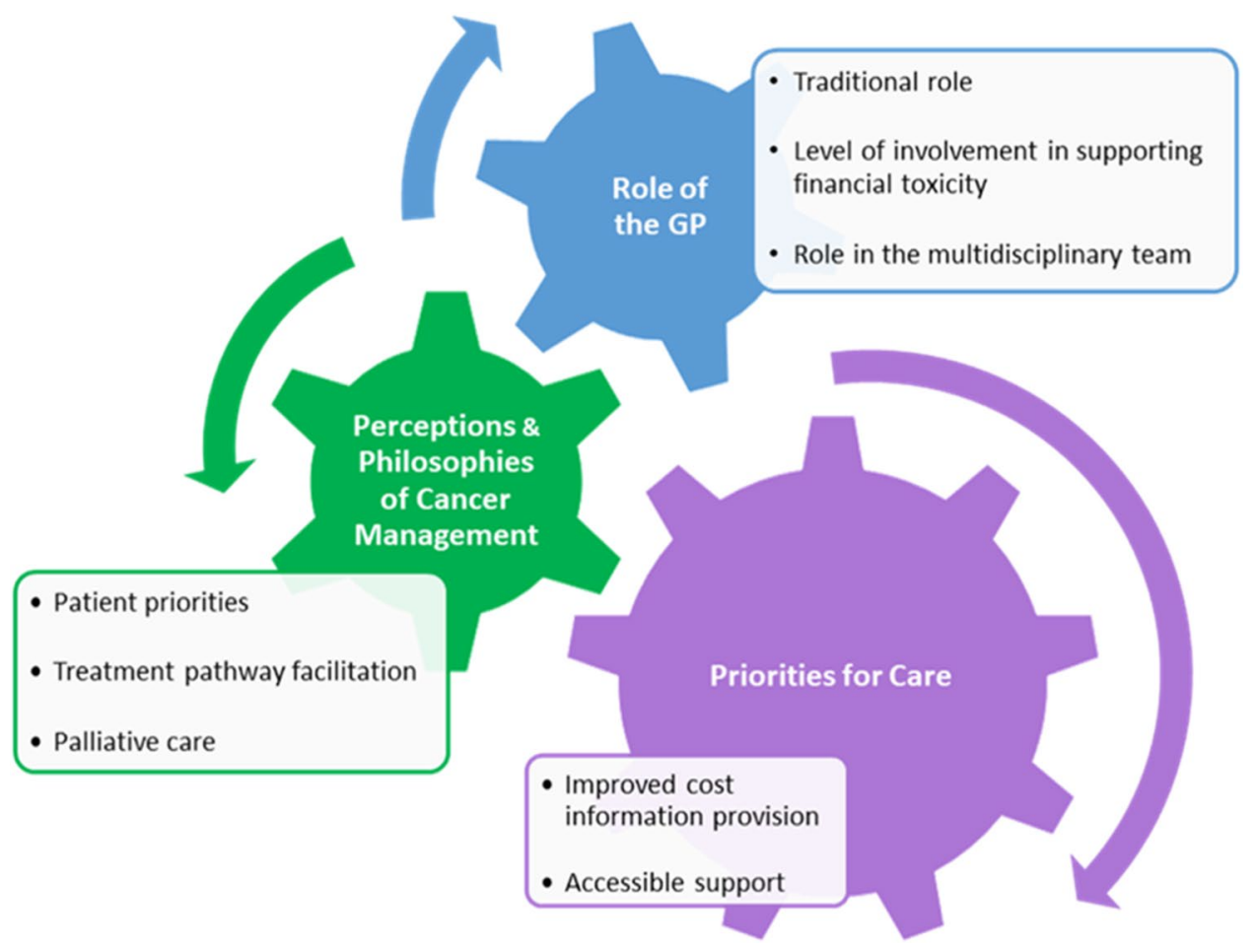

Fig. 1 Graphical representation of themes and subthemes

GPs identified challenges in meeting this role. These challenges were particularly related to their knowledge of diagnostic, treatment, and specialist costings. 'This was related to the fact that 'anyone can charge anything' (GP17):

I unfortunately wouldn't be informed as to how much out-of-pocket expenses they might have ... Broaching a topic that you have no knowledge about, is tantamount to opening a Pandora's Box. (GP14)

This was presented partially as a system barrier but also not necessarily the responsibility of the GP to provide indepth counselling in relation to costs that were essentially out of their control. 'I don't see it as my role to actually ... find financial solutions or to counsel them through those sorts of costs in detail' (GP13).

GPs also highlighted that, while for some cancers managing financial concerns may be achievable, the complexity and heterogeneity of cancer care made it a challenge to address FT:

For prostate, breast, melanoma even, we do have a sense of what that [pathway] might look like, but for cancers which are less common I think it is harder for us to know ... to give meaningful financial counselling. (GP4)

There were also questions about whose role it might be in this setting. 'The ideal thing would be to have a permanent general practice social worker who came and worked in the practice' (GP2). Practice nurses, depending on experience, were also identified as having a role.

\section{Role in the multidisciplinary team (MDT)}

One of the significant barriers to GPs addressing FT beyond the diagnostic phase was the limited communication and collaboration with the specialist teams, including access to survivorship plans:

We're often not involved in a multidisciplinary team discussion ... It's often not very transparent ... surrounding cost; so how long will the care occur for, and what the journey would often look like for a patient.

(GP4)

The disconnect between the GP and the treating team impacted the established and trusted GP-patient relationship. This relationship is important for managing issues such as FT. 'It can cause them to often get a bit disengaged from the GPs. It's one of the significant issues' (GP9).

\section{Perceptions and philosophies of cancer management}

Conversations relating to FT were driven by perceptions of the needs of cancer patients and philosophies about how cancer care should be managed. 


\section{Patient priorities}

GPs believed that at diagnosis, their primary role was to facilitate an appropriate pathway for patients with cancer. In this setting, cost implications and discussions were often guided by perceptions of patient's priorities:

I would say, Look. This is going to be expensive ... More often than not they would just say, "Look. I don't care. Our priority is this, getting this sorted, getting me back on track, getting me back to work and getting me back into my family life" (GP2).

One of the challenges that GPs identified in relation to the prioritisation of quick access to care is the vulnerability of people. This is related to the acuity of cancer leading to an urgency in decision-making which often leads to costly choices. 'They've been given this word called "cancer" ... a lot of the rational thinking goes out the window' (GP11). The impact of FT was perceived by most GPs as something that became an issue later in the cancer trajectory.

\section{Treatment pathway facilitation}

Beyond their broad role as a financial advocate, GPs varied in the way they counselled their patients in making care pathway decisions. On one hand, they saw their role as information provider. On the other, they felt their experience and local knowledge put them in the position to steer a patient in a certain direction. Conversations were also often driven by private health insurance and a perception that people with a high socioeconomic status had a low risk of FT. 'I have to confess here ... I work in a very affluent area, and the costs of whatever care is required, is usually not discussed' (GP14).

Discussion was also driven by the GP's own experience and philosophies related to each type of healthcare system. These philosophies varied from 'The public system is a little bit poor in providing access to novel treatments ... (GP5) to ... I saw that patients were getting the same treatment ... But when they were private, they had a much bigger bill' (GP8). GPs who had recent experience working in tertiary public hospitals often saw public as the best option for patients with cancer due to their multidisciplinary approach to care.

\section{Palliative care}

Perceptions and philosophies changed when discussions turned to palliative care. GPs identified that patients and family expectations change over time, and this may contain costs: '.. they're not going down every rabbit hole looking for a cure ... cost tends to be a lot more contained' (GP11). GPs' philosophies also changed when the goal of care shifted away from cure. Most GPs changed their billing structure, as this was seen as their 'civic duty':

I've never charged a patient to undertake palliative care, that includes going to their homes which can be some distance away ... My personal philosophy is that someone that's dying from cancer should not be given a bill ... (GP1)

\section{Priorities for care}

Training and education and accessible support were seen as priorities to better facilitate FT support by GPs.

\section{Improved cost information provision}

GPs identified that information about costs was learnt 'along the way' and from patient-reported experiences. Many felt that additional cost information would improve their knowledge as specialists did not always provide clear information about this:

I think it probably would be interesting to have a bit more knowledge when people are trying to make those early decisions about if they go private, what are the costs to be expected. (GP6)

Many GPs were not aware of the scope of not-for-profit cancer organisations and their ability to help patients and practitioners. Further, the information and training provided by cancer organisations were considered biomedically driven rather than focusing on psychosocial concerns which would be useful ' ... maybe Cancer Australia needs to not talk about cancer and treatment of the cancer but have an awareness week about financial toxicity' (GP20). Primary Health Networks (PHN) were identified as ideally situated to help disseminate local resource and health pathway information.

\section{Accessible support}

GPs identified that having a thorough cost repository (centralised source of costing data) was unlikely to be achievable but agreed that an online openly accessible resource relating to pathways and locally available resources and support services would be beneficial. Alternatively, some GPs suggested that a telephone advice line would be useful to guide decision-making and to support FT when it arose in the clinic:

This is where being able to actually give GPs a tool guide where someone with cancer goes, "I' $m$ really struggling," ... If you've got issues particularly with financial toxicity. Let's just review what things you can do. (GP20) 
Although not all GPs used telehealth, most who had been exposed to it offered it as an avenue for collaboration with cancer care teams. Furthermore, they felt that government support did not equate with the level of expectation imposed on GPs in supporting cancer care. GPs identified that more Medicare funding was needed to alleviate the financial burden imposed on patients to complete complex health forms and support survivorship initiatives:

With survivorship we're all going down the pathway of promoting those lifestyle things ... there needs to be either more item numbers specific to cancer survivorship ... the government and the health department should be supporting that for the community. (GP9)

\section{Discussion}

Many GPs felt they have the expertise to play a role in supporting FT in cancer patients in our study, but there are limitations to the extent of this role. These limitations include knowledge of cost, complexity of cancer care, role in the MDT, and varying perceptions of health service and care provision. Access to cost information and support must be priorities if GPs are to adequately address FT in cancer patients.

The traditional role of the GP, which is focused on person centredness, comprehensiveness, continuity, trusted, and enduring patient relationships [18], positions them well to play a role in supporting the financial burden related to cancer. Similar to other shared care studies, GPs in this study felt that they played a definitive role in diagnosing and initiating treatment pathways for cancer patients [22]. These time points are pivotal in the FT related to cancer care. It is therefore important for GPs to be well-informed to practice as a partner with the patient when navigating cost-appropriate decisions at such a vulnerable time [23]. Communication related to cost of care has become not only an indicator of quality care but an expectation in Australia [23]. However, informed choice needs to include more than costs charged for diagnostics and individual practitioners. It should account for variations of costs charged for the same service $[16,23]$. This was recognised as a challenge in a system where charges for services are provider-driven.

Australia's healthcare system is a multifaceted mix of public and private providers funded by various governments as well private health insurers and individuals [24]. Philosophies about private and public cancer care influenced the way care pathway choices were offered, with perceptions of these services varying significantly. A driver of these conversations was often private health insurance and GPs' perception of patient wealth. Perceptions from some GPs that people from affluent areas did not have financial concerns related to cancer are not consistent with the literature. Numerous studies have identified that often patients, who at the onset of a cancer diagnosis, can afford cancer care may still experience FT relating to decreases in savings and assets, and an inability to return to work after treatment $[9,15,25]$. It is important that up-to-date data about service wait times and outcomes are clear, so GPs can facilitate patients' informed treatment decision-making. This ability to support patient autonomy in decision-making about diagnostic and treatment pathways is an important part of informed financial consent and is a core role of the GP. Currow and Aranda (2016) highlight that cost disclosure must not only include identifying the cost of procedures or treatment, but also offer alternatives that may be less expensive but have similar treatment outcomes [16]. Although GPs acknowledged that, logistically and pragmatically, a thorough cost repository was unlikely to be achievable; transparent costings of services are essential to give good information and to empower patients to ask questions about costs.

Cost-related health literacy of healthcare professionals has been recognised as a barrier to supporting FT. A small study of cost-related health literacy of oncologists found very few were aware of the out-of-pocket cost of tests and treatments and that this significantly limited their ability to address financial burden with patients [26]. Zafar and colleagues (2015) also recognised barriers to cost-related health literacy are multifactorial and come from an organisational (cost transparency, screening, timely advice), interpersonal (discomfort around discussing cost), and individual level (limited knowledge). Policy changes need to intervene at all three levels through improving cost transparency, the use of screening tools, early intervention, and support for robust and knowledgeable cost conversations by healthcare professionals $[27,28]$. Our study identified that easily accessible information about financial support in the form of websites or telephone lines and promotion of currently available resources is a priority to support GPs in addressing FT.

GPs in this study also recognised that while they play a role in giving broad financial advice, they proposed that other professionals such as social workers or general practice nurses may be better suited to this role. Studies from the USA have identified a role for financial navigators to support the complex nature of FT in cancer care [29]. This role moves beyond counselling and advocacy to work proactively with patients to help them understand the complex nature of insurance and other financial supports along with minimising out-of-pocket expenses without compromising treatment outcomes [29]. Further investigation is required regarding these roles in the Australian context given the significant differences between the two healthcare systems.

A significant barrier to GPs supporting FT in the postdiagnostic phase of cancer care is the lack of communication from the specialist team regarding treatment pathways and 
ongoing care needs. This disconnect directly impacts the ability of GPs to provide financial support to their patients. A systematic review by Lisy et al. (2021) identified communication as a key component for shared care to be successful [30]. Cancer survivorship plans have been identified as an opportunity to jointly disseminate information to patients and their GPs throughout their cancer journey [7]. These plans can include expected costs and indirect costs while on treatment and throughout follow-up care [7]. Yet it was identified by GPs in this study that they saw little or no cancer survivorship care plans from specialist teams. Telehealth, introduced during the COVID-19 pandemic, was seen as an opportunity to improve engagement between the primary and specialist care settings [31]. A report from Cancer Australia recognised the use of telehealth enabled better interaction between patients and their healthcare providers [32]. Further to this, the use of virtual MDT meetings highlighted an opportunity for GPs to connect with the specialist team [32]. This improved ability to collaborate is essential for GPs to understand the overall treatment goals and the financial burden that is being imposed on their patients.

\section{Strengths and limitations}

To our knowledge, this is the first study that has investigated the role of the GP in addressing FT in cancer patients. Participant interest, location of practice, and experience with cancer patients were varied to purposefully generate a broad perspective on the topic, although it is recognised that some unique perspectives may not be represented. Despite this limitation this study provides valuable insights into the role of the GP in addressing FT.

\section{Conclusion}

Financial toxicity in cancer patients can be addressed outside of the specialist cancer setting. Our results have implications for cancer healthcare policy. While solutions to manage FT associated with a cancer diagnosis, its subsequent treatment, and survivorship trajectory require a multidisciplinary approach, it is suggested that the GP can play an important role as part of the team. To do this, improved cost transparency and increased cost health literacy by all healthcare professionals caring for cancer patients are essential. Further, improved communication and collaboration between the specialist teams and GPs can assist the GP to address FT beyond diagnosis and pathway referral. Telehealth and better use of survivorship care plans may facilitate this. Easily accessible information about financial support and promotion of currently available resources is a priority to support GPs in addressing FT.
Acknowledgements The authors gratefully acknowledge support from the Primary Care Collaborative Cancer Clinical Trials Group (PC4), Australia.

Author contribution All authors contributed to the study conception and design. Preparation, data collection, and analysis were performed by CT, JF, and RJC. The first draft of the manuscript was written by $\mathrm{CT}$, and all authors read, commented, and approved various versions and the final manuscript.

Funding CT was supported with a training grant from the Primary Care Collaborative Cancer Clinical Trials Group (PC4), Australia.

RJC receives salary support from the National Health and Medical Research Council through an Investigator Grant (APP1194051).

Availability of data and material Not applicable.

Code availability Not applicable.

\section{Declarations}

Ethics approval This study was approved by the Queensland University of Technology human research ethics committee (1900000798).

Consent to participate Verbal informed consent was obtained prior to the interview.

Consent for publication The authors affirm that human research participants provided informed consent for publication.

Conflict of interest The authors declare no competing interests.

\section{References}

1. Carrera PM, Kantarjian HM, Blinder VS (2018) The financial burden and distress of patients with cancer: understanding and stepping-up action on the financial toxicity of cancer treatment. CA Cancer J Clin 68(2):153-165. https://doi.org/10.3322/caac. 21443

2. Desai A, Gyawali B (2020) Financial toxicity of cancer treatment: moving the discussion from acknowledgement of the problem to identifying solutions. EClinicalMedicine 20:100269. https://doi. org/10.1016/j.eclinm.2020.100269

3. Kayser K, Smith L, Washington A, Harris LM, Head B (2020) Living with the financial consequences of cancer: a life course perspective. J Psychosoc Oncol 1-18. https://doi.org/10.1080/ 07347332.2020 .1814933

4. Lentz R, Benson AB 3rd, Kircher S (2019) Financial toxicity in cancer care: prevalence, causes, consequences, and reduction strategies. J Surg Oncol 120(1):85-92. https://doi.org/10.1002/ jso. 25374

5. Pearce A, Tomalin B, Kaambwa B, Horevoorts N, Duijts S, Mols F, van de Poll-Franse L, Koczwara B (2019) Financial toxicity is more than costs of care: the relationship between employment and financial toxicity in long-term cancer survivors. J Cancer Surviv 13(1):10-20. https://doi.org/10.1007/s11764-018-0723-7

6. Zhu Z, Xing W, Zhang X, Hu Y, So WKW (2020) Cancer survivors' experiences with financial toxicity: a systematic review and meta-synthesis of qualitative studies. Psychooncology 29(6):945959. https://doi.org/10.1002/pon.5361 
7. Coughlin SS, Dean LT (2019) Cancer survivorship care plans, financial toxicity, and financial planning alleviating financial distress among cancer survivors. Support Care Cancer 27(6):19691971. https://doi.org/10.1007/s00520-019-04703-6

8. Longo CJ, Fitch MI, Banfield L, Hanly P, Yabroff KR, Sharp L (2020) Financial toxicity associated with a cancer diagnosis in publicly funded healthcare countries: a systematic review. Support Care Cancer 28(10):4645-4665. https://doi.org/10.1007/ s00520-020-05620-9

9. Gordon LG, Merollini KMD, Lowe A, Chan RJ (2017) A systematic review of financial toxicity among cancer survivors: we can't pay the co-pay. Patient 10(3):295-309. https://doi.org/10.1007/ s40271-016-0204-x

10. Kale HP, Carroll NV (2016) Self-reported financial burden of cancer care and its effect on physical and mental health-related quality of life among US cancer survivors. Cancer 122(8):283-289. https://doi.org/10.1002/cncr.29808

11. Khera N, Chang YH, Hashmi S, Slack J, Beebe T, Roy V, Noel P, Fauble V, Sproat L, Tilburt J, Leis JF, Mikhael J (2014) Financial burden in recipients of allogeneic hematopoietic cell transplantation. Biol Blood Marrow Transplant 20(9):1375-1381. https://doi. org/10.1016/j.bbmt.2014.05.011

12. Sharp L, Timmons A (2016) Pre-diagnosis employment status and financial circumstances predict cancer-related financial stress and strain among breast and prostate cancer survivors. Supportive Care Cancer 24(2):699-709. https://doi.org/10.1007/ s00520-015-2832-4

13. Catalano PJ, Ayanian JZ, Weeks JC, Kahn KL, Landrum MB, Zaslavsky AM, Lee J, Pendergast J, Harrington DP, Cancer Care Outcomes Research Surveillance C (2013) Representativeness of participants in the cancer care outcomes research and surveillance consortium relative to the surveillance, epidemiology, and end results program. Med Care 51(2):e9-15. https://doi.org/10.1097/ MLR.0b013e318222a711

14. Fenn KM, Evans SB, McCorkle R, DiGiovanna MP, Pusztai L, Sanft T, Hofstatter EW, Killelea BK, Knobf MT, Lannin DR, Abu-Khalaf M, Horowitz NR, Chagpar AB (2014) Impact of financial burden of cancer on survivors' quality of life. J Oncol Pract 10(5):332-338. https://doi.org/10.1200/JOP.2013.001322

15. Koczwara B (2017) Unemployment after cancer - a hidden driver of financial toxicity. Cancer Forum 41(2):36-41

16. Currow D, Aranda S (2016) Financial toxicity in clinical care today: a "menu without prices"1. Med J Aust 204(11):397-397. https://doi.org/10.5694/mja16.00182

17. Chan R, Gordon L, Zafar S, Miaskowski C (2018) Financial toxicity and symptom burden: what is the big deal? Support Care Cancer Inpress. https://doi.org/10.1007/s00520-018-4092-6

18. World Health Organisation (2008) The World Health Report 2008: primary health care (now more than ever). https://www.who.int/ whr/2008/en/. Accessed 7th Oct 2020

19. Rubin G, Berendsen A, Crawford SM, Dommett R, Earle C, Emery J, Fahey T, Grassi L, Grunfeld E, Gupta S, Hamilton W, Hiom S, Hunter D, Lyratzopoulos G, Macleod U, Mason R,
Mitchell G, Neal RD, Peake M, Roland M, Seifert B, Sisler J, Sussman J, Taplin S, Vedsted P, Voruganti T, Walter F, Wardle J, Watson E, Weller D, Wender R, Whelan J, Whitlock J, Wilkinson C, de Wit N, Zimmermann C (2015) The expanding role of primary care in cancer control. Lancet Oncol 16(12):1231. https:// doi.org/10.1016/S1470-2045(15)00205-3

20. Charmaz K (2014) Constructing grounded theory. SAGE, London

21. Silverman D (2017) Doing qualitative research, 5 end. SAGE, London

22. Mitchell GK, Burridge LH, Colquist SP, Love A (2012) General practitioners' perceptions of their role in cancer care and factors which influence this role. Health Soc Care Community 20(6):607616. https://doi.org/10.1111/j.1365-2524.2012.01075.x

23. Australian Medical Association (2020) Informed financial consent: a collaboration between doctors and patients. AMA

24. Australian Institute of Health Welfare (2020) Health system overview. AIHW. https://www.aihw.gov.au/reports/australias-health/ health-system-overview. Accessed 10 Feb 2021

25. Thomas T, Hughes T, Mady L, Belcher SM (2019) Financial toxicity: a review of the literature and nursing opportunities. Clin J Oncol Nurs 23(5):5-13. https://doi.org/10.1188/19.CJON.S2.5-13

26. Fischer KA, Walling A, Wenger N, Glaspy J (2020) Cost health literacy as a physician skill-set: the relationship between oncologist reported knowledge and engagement with patients on financial toxicity. Support Care Cancer. https://doi.org/10.1007/ s00520-020-05406-Z

27 Zafar SY (2016) Financial toxicity of cancer care: it's time to intervene. J Natl Cancer Inst 108(5):370. https://doi.org/10.1093/ jnci/djv370

28. Gordon LG, Chan RJ (2017) Financial toxicity among patients with cancer-where to from here? Cancer Nurs 40(4):257-258. https://doi.org/10.1097/NCC.0000000000000510

29. Sherman D, Fessele KL (2019) Financial support models: a case for use of financial navigators in the oncology setting. Clin J Oncol Nurs 23(5):14-18. https://doi.org/10.1188/19.CJON.S2. 14-18

30. Lisy K, Kent J, Piper A, Jefford M (2021) Facilitators and barriers to shared primary and specialist cancer care: a systematic review. Support Care Cancer 29(1):85-96. https://doi.org/10. 1007/s00520-020-05624-5

31. Chan A, Ashbury F, Fitch MI, Koczwara B, Chan RJ (2020) Cancer survivorship care during COVID-19-perspectives and recommendations from the MASCC survivorship study group. Support Care Cancer 28(8):3485-3488. https://doi.org/10.1007/ s00520-020-05544-4

32. Cancer Australia (2020) COVID-19 recovery: implications for cancer care

Publisher's note Springer Nature remains neutral with regard to jurisdictional claims in published maps and institutional affiliations. 